\title{
EVIDENCE OF CONFINEMENT OF SOLAR-ENERGETIC PARTICLES TO INTERPLANETARY MAGNETIC FIELD LINES
}

\author{
E. E. Chollet ${ }^{1,2}$ and J. Giacalone ${ }^{2}$ \\ ${ }^{1}$ California Institute of Technology, Pasadena, CA 91125, USA \\ ${ }^{2}$ Lunar and Planetary Laboratory, University of Arizona, Tucson, AZ 85721, USA \\ Received 2010 April 5; accepted 2010 December 8; published 2011 January 20
}

\begin{abstract}
We present new observations of solar-energetic particles (SEPs) associated with impulsive solar flares that show evidence for their confinement to interplanetary magnetic field lines. Some SEP events exhibit intermittent intensity dropouts because magnetic field lines filled with and empty of particle flux mix together. The edges of these dropouts are observed to be very sharp, suggesting that particles cannot easily move from a filled to an empty field line in the time available during their transport from the Sun. In this paper, we perform high time-resolution observations of intensity fall-off at the edges of observed SEP dropouts in order to look for signatures of particle motion off field lines. However, the statistical study is dominated by one particularly intense event. The inferred length scale of the intensity decay is comparable to the gyroradii of the particles, suggesting that particles only rarely scatter off magnetic field lines during interplanetary transport.
\end{abstract}

Key words: diffusion - Sun: activity - Sun: particle emission

\section{INTRODUCTION}

Solar activity, in the forms of flares and coronal mass ejections (CMEs), is one of the most efficient particle accelerators known. Within just a few seconds, particles are accelerated to substantial fractions of the speed of light, subsequently traveling out through the solar system and reaching Earth in just a few hours. Solar-energetic particles (SEPs) accelerated close to the Sun often exhibit velocity dispersion when observed at $1 \mathrm{AU}$, with the higher energy, higher speed particles arriving first, and the lower energy, slower speed particles arriving later. An example is shown in Figure 1: see Section 2 for a discussion of these observations. The earliest arriving particles at each energy travel with very small pitch angles to the magnetic field and no scattering, so they suffer very little adiabatic cooling. Since their speed remains approximately constant, the time they were injected onto the field line connecting the source and the spacecraft can be easily estimated, and the particle arrival time as a function of energy can be used to estimate their travel distance.

The particles' transport through the solar system is guided by the interplanetary magnetic field that is convected outward by the solar wind plasma. Particles can move from one field line to adjacent field lines through gradient and curvature drifts or through particle scattering off magnetic irregularities on the scale of their gyroradii. However, in the inner solar system, the drift velocities of the low-rigidity particles considered in this study (rigidity on the order of a hundred MV) are only a fraction of the solar wind speed (Jokipii et al. 1977), limiting the distance the particles can drift, and the differences in the drift motions of different individual particles are very small. By contrast, particle scattering is a stochastic process, allowing some to move to new field lines and some to remain on their original field lines.

Numerical and observational studies have shown the particle scattering mean free path to be a substantial fraction of an $\mathrm{AU}$ or more (e.g., Droge et al. 2010; Qin et al. 2004; Palmer 1982). Because the particles experience little scattering, gradients created at the Sun can remain substantially intact during travel through the inner heliosphere, where they are observed in situ. As a spacecraft crosses field lines that are alternatively connected and not connected to an ion source in an impulsive flare-related event, it sees intermittent, dispersionless intensity "dropouts," as reported by Mazur et al. (2000) and Giacalone et al. (2000). These dropouts are readily seen in Figure 1. However, some scattering does still occur, in principle allowing particles to leak onto empty field lines in the intensity dropouts. The purpose of this paper is to study the edges of these dropouts to determine the relevant temporal and spatial scales associated with this motion across the local field.

In this work, we present an analysis of high time-resolution observations of dropout edges with a focus on determining whether this transport across the local field can be detected during Sun-Earth travel. In the following section, we summarize the observations, and in Section 3 we show how these observations demonstrate that particles are strongly tied to field lines.

\section{OBSERVATIONS}

The energetic ion data used here are from the Ultra Low Energy Isotope Spectrometer (ULEIS: Mason et al. 1998) on board the Advanced Composition Explorer (ACE: Stone et al. 1998) spacecraft, and these include the species carbon through iron with energies approximately between 0.2 and $10 \mathrm{MeV}$ nucleon $^{-1}$. Chollet \& Giacalone (2008) compiled a list of impulsive-flare-related SEP events based on their composition and evidence of the speed dispersion produced by particle transport. We examined all the dropouts in Table 1 of their paper and chose edges with a sufficient number of particles to produce good statistics, defined as 25 or more particles in a bin of a particular width (see below) and above a minimum energy threshold. The ULEIS instrument includes two detectors with overlapping energy ranges but differing geometry factors, so we excluded particles below $0.2 \mathrm{MeV}$ nucleon $^{-1}$ to only include the particles observed solely by the high-energy detector. However, some events had significant background above $0.2 \mathrm{MeV}$ nucleon ${ }^{-1}$, so we used a higher minimum energy threshold for those events.

ULEIS only observes particles with energies below $10 \mathrm{MeV}$ nucleon $^{-1}$, so the speed range of the particles in this study is very small. The particle speeds range from $0.14 \mathrm{AU} \mathrm{hr}^{-1}$ to 


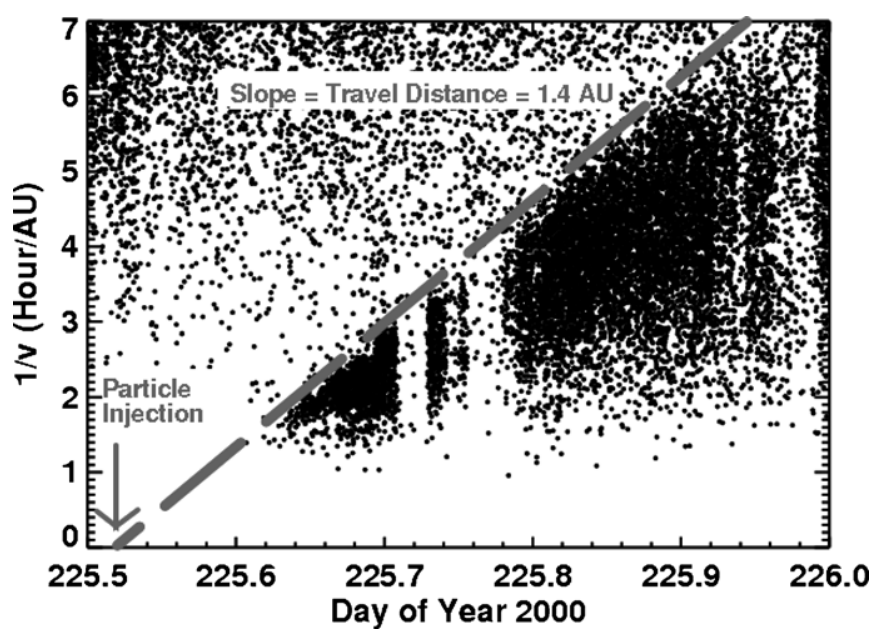

Figure 1. Example of SEP event, with each dot representing an ion of a particular energy observed at a particular time. The velocity dispersion is clear, with the high-energy, high-velocity particles at the bottom and the low-energy, lowvelocity particles at the top. The fit to the dispersion, given in gray, gives the distance the particles travel as well as their injection time.

$1 \mathrm{AU} \mathrm{hr}^{-1}$, so the lowest energy particles travel the Sun-Earth distance in $\sim 6 \mathrm{hr}$ and the highest energy particles travel the Sun-Earth distance in $\sim 1 \mathrm{hr}$. When this limited particle mobility is considered along with a well-determined injection time of the earliest arriving particles from X-ray observations of a flare, strong constraints can be set on how much pitch angle scattering can delay these particles' arrival at Earth. At any given energy, the event lasts a few hours, so the latest arriving particles of that energy could only have traveled a fraction of an AU further than the earliest arriving particles. These constraints allow us to study the cross-field transport without having to worry about the different distances different individual particles have traveled due to pitch angle scattering.

Each of these events consisted of one or more velocity dispersions, but, in some cases, a single event includes several particle dispersions which overlap in time, arising from multiple closely spaced injections onto field lines at the Sun. We eliminated edges that crossed several speed dispersions, since the field line may be connected to one particle injection source but not another, creating multiple edges at slightly different times that appear to be one edge. A minority of dropouts is known to be caused by a large shift in magnetic connection location at the Sun due to boundaries in the solar wind, such as shocks or current sheets (Gosling et al. 2004). With such a large shift, the field lines are not adjacent during most of the travel time of the particles, so the particles do not have as much time to move from one field line to the next. Thus, we did not include events with large connection shifts in this study.

The ULEIS data used here list particle arrival time, energy, and mass, so we converted time to distance units to be able to determine the distance that particles travel across the field. A schematic of how the conversion from time to distance units was performed is presented in Figure 2. The spacecraft is stationary with the solar wind blowing past it radially, and the magnetic field is at some angle to the solar wind convection. We found the average magnetic field direction and solar wind speed for each event, then took the cross-field component of the velocity to determine the length of time it takes a particular fixedwidth bin to move past the spacecraft. We then summed the number of particles in each 50,000 km wide bin for our analysis.
Table 1

Events Used in This Study

\begin{tabular}{cccccc}
\hline \hline Year & DOY & $E_{\text {threshold }}$ & $|B|(\mathrm{nT})$ & B angle $^{\text {a }}$ & B angle r.m.s. \\
\hline 1998 & 130.216 & 0.3 & 5 & 66 & 33 \\
1999 & 220.102 & 0.2 & 7 & 24 & 18 \\
2000 & 123.035 & 0.2 & 10 & 55 & 30 \\
2000 & 225.707 & 0.3 & 20 & 19 & 14 \\
2000 & 225.731 & 0.3 & 20 & 19 & 14 \\
2000 & 225.738 & 0.3 & 20 & 19 & 14 \\
2000 & 225.790 & 0.3 & 20 & 19 & 14 \\
2000 & 225.962 & 0.3 & 20 & 19 & 14 \\
2000 & 225.993 & 0.3 & 20 & 19 & 14 \\
2002 & 270.875 & 0.2 & 10 & 25 & 12 \\
2002 & 270.933 & 0.2 & 10 & 25 & 20 \\
2003 & 273.832 & 0.2 & 6 & 55 & \\
\hline
\end{tabular}

Note. ${ }^{a}$ Angle between the solar wind velocity (radial) and the cross-field component, equivalent to angle $a$ in Figure 2.

Some dropouts, when converted to distance units, became very narrow, such that the increasing and decreasing intensity edges overlapped. Any edge that had another edge within five bins was eliminated from the sample. Table 1 lists all the dropout edges that remained after all these criteria were applied.

Because these events are typically low intensity, superpositions of multiple events are necessary to overcome the poor statistics of individual edges. To make each superposition, we took an average of the counts in bins outside the dropout for each edge, then lined up the bins that first exceeded those averages. To account for different amounts of scattering predicted by different diffusion theories, we performed the superpositions as follows.

1. A simple superposition of all events.

2. Separate superpositions of high-mass (greater than $20 \mathrm{amu}$ ) and low-mass (12-20 amu) particles. Since the mass-tocharge ratio of carbon, nitrogen, and oxygen should be similar to each other but different from iron, and these species make up the bulk of the particles observed, the split at 20 amu should show any mass-to-charge dependence of the amount of scattering.

3. Separate superpositions of events that occurred inside and outside interplanetary coronal mass ejections (ICMEs). Various measurements of the length of magnetic field lines making up ICMEs give 2-3 AU (Larson et al. 1997; Chollet et al. 2007), while the standard Parker spiral magnetic field lines are 1.1 AU in length. Particles traveling on ICME field lines must then travel two to three times as far as those outside ICMEs, changing the amount of time they have to move to empty field lines. In addition, the variance in the magnetic field is typically low in ICMEs, decreasing the amount of scattering of particles inside them (Torsti et al. 2004).

We fit each edge (overplotted in gray) using a maximum likelihood method assuming Gaussian uncertainties and an exponential model with two unknown parameters, i.e., $N(x)=$ $A \exp (-x / L)$, where $x$ is the distance from the edge and $N(x)$ is the number of particles in the bin. The background number of particles at the center of each dropout was similar to the background level before and after each event. We subtracted off the background (found by a mean of points at the center of the dropout corresponding to each edge) before fitting. However, the uncertainties are likely non-Gaussian since there are only a 


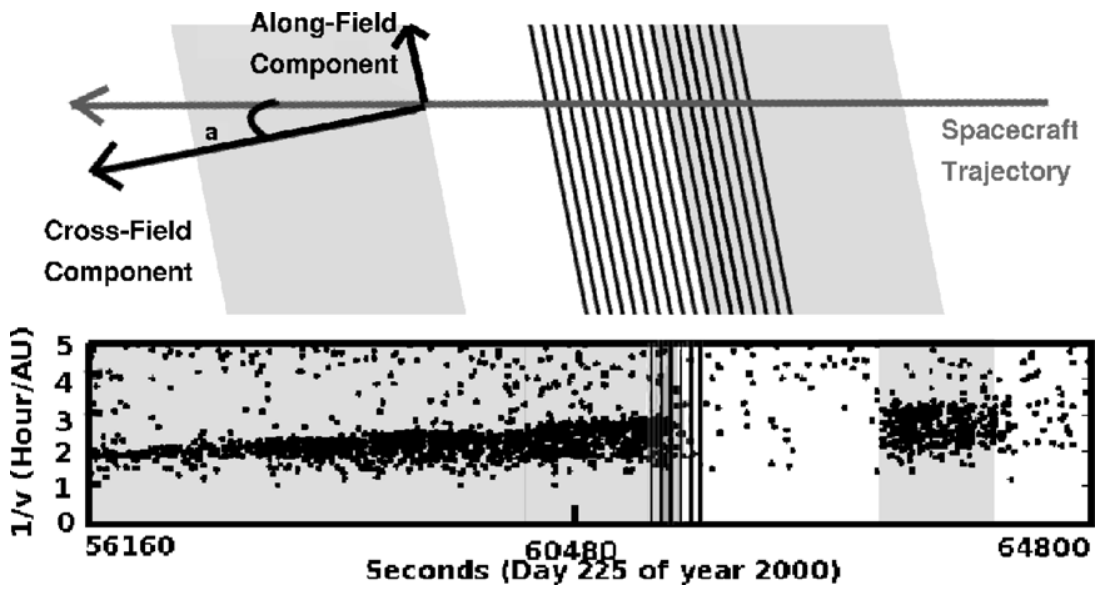

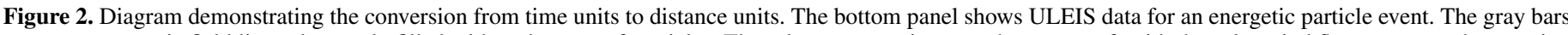

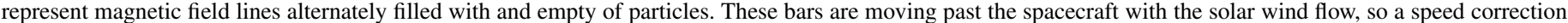

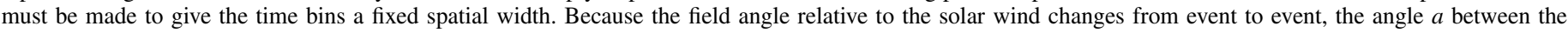
spacecraft trajectory and the cross-field component is computed for each event individually.

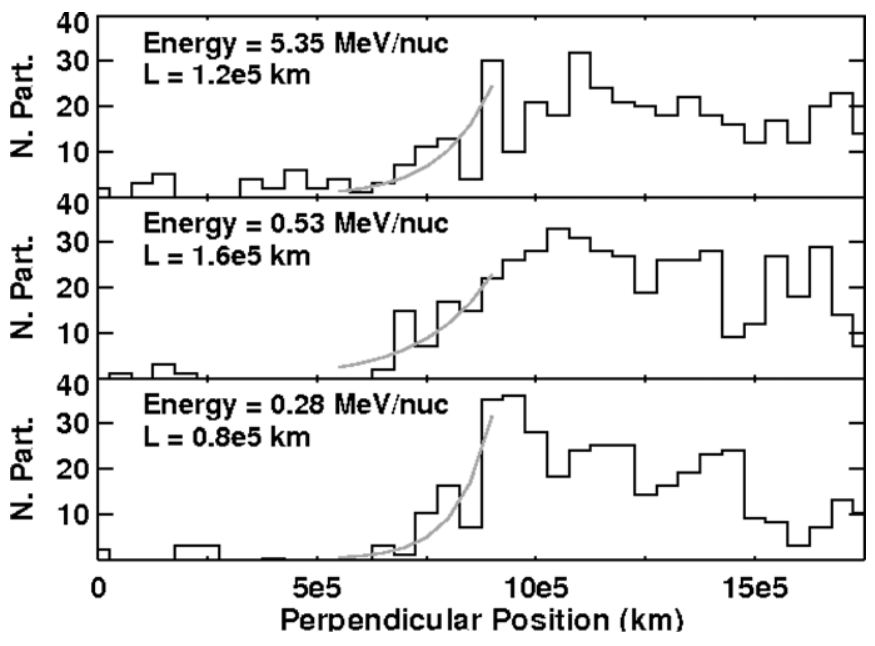

Figure 3. Superposition of all events not in an ICME, split into three energy bins. The average bin energy and measured diffusion length (from the fit in gray) are printed in each bin. Each bin contains very few particles, and the measured diffusion length depends strongly on which bins are chosen for the fit.

few counts in some of the bins. Choosing a somewhat different bin range for the fit changes the measured length by an amount on the order of the measured length, so the uncertainty from the bin choice dominates the statistical uncertainties given by the method. The lengths measured here are more of an order-ofmagnitude estimate rather than a precise value.

\section{ANALYSIS}

Superpositions using particle mass and the presence of ICMEs, as described above, are hampered by poor statistics. When we use purely events with no ICMEs, after background subtraction, no bin has more than 40 counts (Figure 3 ). When we superpose high mass and low mass separately, the total count in each bin drops to half its previous value, and the low counts make it more difficult to separate the background and event intensity. Since the dropout edge needs to be at least several bins wide to fit it to the function, using wider bins is not practical for this study. For events with a Parker spiral field and a solar wind speed of $400 \mathrm{~km} \mathrm{~s}^{-1}$, the $50,000 \mathrm{~km}$ bin corresponds to a time bin of

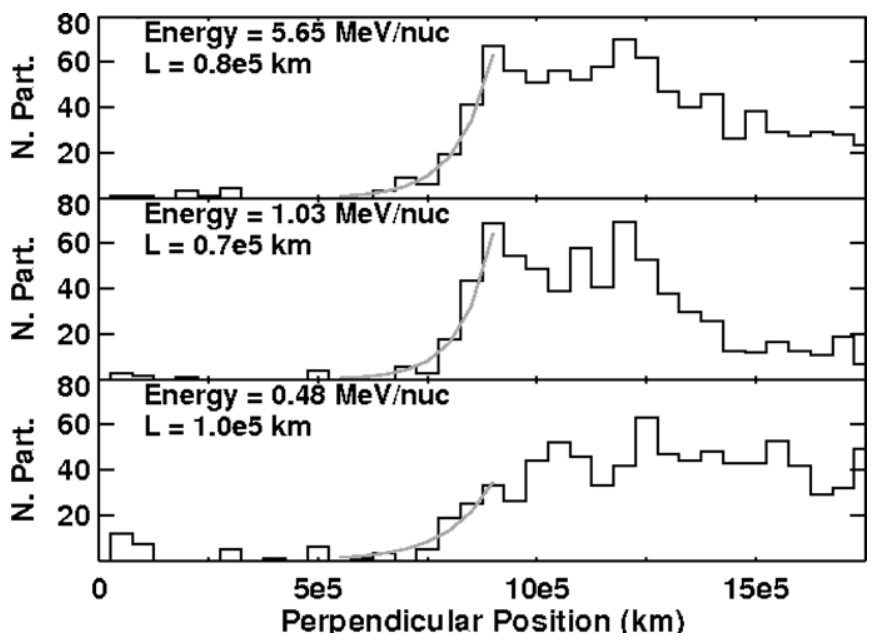

Figure 4. Superposition of the six edges making up the 2000/225 energetic particle event, split into three energy bins. The average bin energy and measured diffusion length (from the fit in gray) are printed in each bin.

roughly $180 \mathrm{~s}$, while the instrument cadence is only $12 \mathrm{~s}$. Since flare-related events are of low intensity, each $12 \mathrm{~s}$ instrument interval will typically have only a few particles. More events or more intense events would ease this problem, and, as the next solar cycle progresses, adding more events to this study would be valuable.

Because of very poor statistics on most events, the simple superposition of all events regardless of ICMEs or particle species is dominated by the six edges from one particular event in 2000, Day of Year (DOY) 225 (the ULEIS data and trajectory fit are in Figure 1, and the cross-field fits are in Figure 4). The particles from this event make up slightly less than half of the total simple superposition. The inferred injection time of this event corresponds to an M1.1 flare observed in X-rays by the GOES spacecraft at 10:09 UT, and the highest energy particles arrive about $6 \mathrm{hr}$ later, as would be expected for direct Earth-Sun travel over an inferred distance of 1.4 AU. According to the ICME lists of Jian et al. (2006) and Cane \& Richardson (2003), this SEP event occurred inside an ICME. This event was also observed by the Wind/STEP particle detector (von 
Rosenvinge et al. 1995), which has the capability to observe particle direction that the ULEIS detector lacks. While no anisotropy measurements are possible early in the event, Wind/ STEP observed 320-640 keV nucleon ${ }^{-1}$ ions to be streaming anti-sunward after 18:00 on DOY 225 (M. Desai 2010, private communication).

While the standard quasilinear theory (Jokipii 1966) would expect more scattering from filled to empty field lines at the high energies, analysis of our data cannot determine if the scattering changes as a function of energy. The 2000/225 event which dominates the superposition occurs inside an ICME, though the results from this event are qualitatively similar to the results from events outside ICMEs (Figure 3). Differing scattering properties inside the ICME (Torsti et al. 2004) or the slight field rotation during the portion of the event with dropouts could be affecting the value of the measured diffusion length. However, neither of these effects should change the dependence on energy, only the absolute measure of the value. Within the precision allowed by this analysis, the distance particles diffuse during Earth-Sun travel is the same at all three energies.

The typical gyroradius of a particle at these energies at $1 \mathrm{AU}$ is of order $10^{4} \mathrm{~km}$, so the measured distances that the particles move from a filled field line listed in Table 1 are only the order of a gyroradius. Since the particle gyroradius increases with the particle energy, the measured length should be larger at higher energies, but even this effect is absent from the 2000/225 event and consequently from the superposition of all events. The particles therefore experience no cross-field motion that can be measured with these data and may only scatter a very few times, if at all, between the Sun and the Earth, suggesting the mean free path for cross-field diffusion by random particle scattering is at least $1 \mathrm{AU}$.

The distance the particles move onto empty field lines as a function of energy does seem to be sensitive to the events chosen. If, for example, the intensity criterion is based on particles per minute rather than particles per kilometer, different events remain in the sample, and different energy dependences can be produced. This sensitivity to sample selection suggests that the energetic particle scattering mean free path may vary from event to event. It may even suggest that the cross-field motion is not easily parameterizable, e.g., by mean free path, or that several competing processes are at work. The events in ICMEs, which dominate the chosen sample, could have a substantially different scattering mean free path in addition to the longer particle travel distance. The particle events are simply not intense enough to be able to distinguish between these possibilities.

\section{CONCLUSIONS}

We analyzed SEP events exhibiting intermittent dropouts in particle intensity due to disconnections from the particle source to study energetic particle motion across the magnetic field. We found that the distance particles move off field lines onto adjacent field lines during travel between the Sun and Earth is on the order of a gyroradius. Our results suggest that energetic particles tend to be strongly tied to field lines, such that almost no cross-field motion occurs between injection at the Sun and 1 AU. We superposed many energetic particle events in an attempt to get a clearer signal, but the superposition of events was dominated by one event from 2000, and that one event probably cannot be generalized to all SEP events. However, trying to exclude particles that should be experiencing different amounts of scattering (such as those inside ICMEs or those with high mass to charge ratios) results in too few particles to fit the dropout edge adequately. No energy dependence of the decay time can be found from these data. More events or higher intensity events are needed to properly measure the cross-field motion of solar-energetic ions. Those events may appear as the solar cycle ramps up, or from other spacecraft in different part of the heliosphere. Closer to the Sun, the particle events are likely to be more intense, making this study a good target for near-Sun missions like Solar Probe.

\section{REFERENCES}

Cane, H. V., \& Richardson, I. G. 2003, J. Geophys. Res., 108, 1156

Chollet, E. E., \& Giacalone, J. 2008, ApJ, 688, 1368

Chollet, E. E., Giacalone, J., Mazur, J. E., \& Al Dayeh, M. 2008, ApJ, 669, 615

Dröge, W., Kartavykh, Y. Y., Klecker, B., \& Kovaltsov, G. A. 2010, ApJ, 709, 912

Giacalone, J., Jokipii, J. R., \& Mazur, J. E. 2000, ApJ, 532, L75

Gosling, J. T., Skoug, R. M., McComas, D. J., \& Mazur, J. E. 2004, ApJ, 614, 412

Jian, L., Russell, C. T., Luhmann, J. G., \& Skoug, R. M. 2006, Sol. Phys., 239, 393

Jokipii, J. R. 1966, ApJ, 146, 480

Jokipii, J. R., Levy, E. H., \& Hubbard, W. B. 1977, ApJ, 213, 861

Larson, D. E., et al. 1997, Geophys. Res. Lett., 24, 1911

Mason, G. M., et al. 1998, Space Sci. Rev., 86, 409

Mazur, J. E., Mason, G. M., Dwyer, J. R., Giacalone, J., Jokipii, J. R., \& Stone, E. C. 2000, ApJ, 532, L79

Palmer, I. D. 1982, Rev. Geophys. Space Phys., 20, 335

Qin, G., Zhang, M., Dwyer, J. R., Rassoul, H. K., \& Mason, G. M. 2005, ApJ, 627,562

Stone, E. C., Frandsen, A. M., Mewaldt, R. A., Christian, E. R., Margolies, D., Ormes, J. F., \& Snow, F. 1998, Space Sci. Rev., 86, 1

Torsti, J., Riihonen, E., \& Kocharov, L. 2004, ApJ, 600, L83

von Rosenvinge, T. T., et al. 1995, Space Sci. Rev., 71, 155 